\title{
Secular spin-down of the AMP XTE J1751-305
}

\author{
A. Riggio ${ }^{1,2}$, L. Burderi ${ }^{2}$, T. Di Salvo ${ }^{3}$, A. Papitto ${ }^{2}$, A. D'Aì ${ }^{3}$, R. Iaria ${ }^{3}$, and M. T. Menna ${ }^{4}$ \\ 1 INAF/Osservatorio Astronomico di Cagliari, località Poggio dei Pini, strada 54, 09012 Capoterra, Italy \\ e-mail: ariggio@oa-cagliari.inaf.it \\ 2 Università di Cagliari, Dipartimento di Fisica, SP Monserrato-Sestu km 0, 7, 09042 Monserrato (CA), Italy \\ 3 Dipartimento di Scienze Fisiche e Astronomiche, Università di Palermo, via Archirafi 36, 90123 Palermo, Italy \\ ${ }^{4}$ Osservatorio Astronomico di Roma, Sede di Monteporzio Catone, via Frascati 33, 00040 Roma, Italy
}

Received 28 April 2010 / Accepted 6 May 2011

\begin{abstract}
Context. Of the 13 known accreting millisecond pulsars, only a few have displayed more than one outburst during the RXTE era. After its main outburst in 2002, XTE J1751-305 showed an additional three dim outbursts. We report on the timing analysis of the latest one, which occurred on October 8, 2009 and was serendipitously observed from its very beginning by RXTE.

Aims. By detecting the pulsation during more than one outburst, we derive a stronger constraint of the orbital parameters and their evolution, and we can track the secular spin frequency evolution of the source.

Methods. Using the RXTE data of the last outburst of the AMP XTE J1751-305, we performed a timing analysis to more accurately constrain the orbital parameters. Because of the low quality of the data statistics, we applied an epoch-folding search technique to the whole data set to improve the local estimate of the time of ascending node passage.

Results. Using this new orbital solution, we epoch-folded data obtaining three pulse phase delays over a time span of 1.2 days, that we fitted using a constant spin frequency model. Comparing this barycentric spin frequency with that of the 2002 outburst, we obtained a secular spin frequency derivative of $-0.55(12) \times 10^{-14} \mathrm{~Hz} \mathrm{~s}^{-1}$. We estimate the pulsar's magnetic dipole value by assuming that the secular spin-down is due to a rotating magneto dipole emission, to be consistent with what is assumed for radio pulsars. We derive an estimate of the magnetic field strength at the polar cap of $B_{\mathrm{PC}}=4.0(4) \times 10^{8} \mathrm{G}$, for a neutron star mass of $1.4 M_{\odot}$, assuming the Friedman Pandharipande Skyrme equation of state.
\end{abstract}

Key words. stars: neutron - stars: magnetic field - pulsars: general - pulsars: individual: XTE J1751-305 - X-rays: binaries

\section{Introduction}

XTE J1751-305 is one of the accretion-powered millisecond $\mathrm{X}$-ray pulsars (AMPs) that displayed more than one outburst in the RXTE era. Recurrent outbursts were also observed in SAX J1808.4-3658 (di Salvo et al. 2008; Burderi et al. 2009; Hartman et al. 2009), IGR J00291+5934 (Galloway et al. 2005, 2008; Patruno 2010; Hartman et al. 2011; Papitto et al. 2010), NGC 6440 X-2 (Altamirano et al. 2010), and Swift J1756.9-2508 (Patruno et al. 2010). XTE J1751-305 was detected for the first time by RXTE on April 3, 2002 (Markwardt et al. 2002, M02, henceforth). This outburst was the brightest and longest of the four displayed by XTE J1751-305, permitting M02 and Papitto et al. (2008) to obtain a full orbital solution.

The second outburst was spotted by INTEGRAL (Grebenev et al. 2005) on March 28, 2005 and lasted 2 days with a peak flux that was $\sim 14 \%$ of the one reached during the first outburst. Unfortunately, the RXTE PCA instrument was not in the proper mode to detect pulsations during the first observation (Swank et al. 2005). The follow-up observations was done in event and single bit modes, but the source had already dimmed below the detection limit. The attribution of this outburst to XTE J1751305 is uncertain, since pulsations were not detected and the INTEGRAL IBIS/ISGRI source position is compatible with the position of at least one other source, IGR J17511-3057 (Papitto et al. 2010; Riggio et al. 2011).

The third outburst was detected on April 5, 2007 by RXTE with a peak flux of $18 \%$ of the first outburst (Markwardt \& Swank 2007; Falanga et al. 2007), very similar to the second outburst. In the subsequent pointed observation by RXTE, the source became too dim to detect pulsations. In this case, the source identification is certain, thanks to a simultaneous Swift observation (Markwardt et al. 2007).

The latest outburst was first spotted with INTEGRAL (Chenevez et al. 2009). Fortunately, it occurred while RXTE was observing the last discovered AMP IGR J17511-3057, very near to XTE J1751-305 (Markwardt et al. 2009). The detection of the $435 \mathrm{~Hz}$ X-ray pulsation and a following Swift observation confirmed that the source in outburst was XTE J1751-305 and not a re-brightening of IGR J17511-3057. In this paper, we report on the timing analysis of this outburst.

\section{Observation and data analysis}

We analysed RXTE PCA observations of XTE J1751-305. We used data from the PCA (proportional counter array, see Jahoda et al. 2006) instrument on board of the RXTE satellite (ObsId 94041 and 94042). We used data collected in event packing mode, with a time and energy resolution of $122 \mu \mathrm{s}$ and 64 energy channels, respectively. Although the XTE J1751-305 pulsation was detected only during observations performed on 8 and 9 October 2009 (Markwardt et al. 2009), the data analysed here cover the time span from 6 October 2009 to 22 October 2009, as shown in Fig. 1. This allowed us to precisely determine any contribution to the observed emission attributable to the AMP IGR J17511-3057, which was still in outburst during the RXTE observation, and to the Galactic ridge. We obtained 


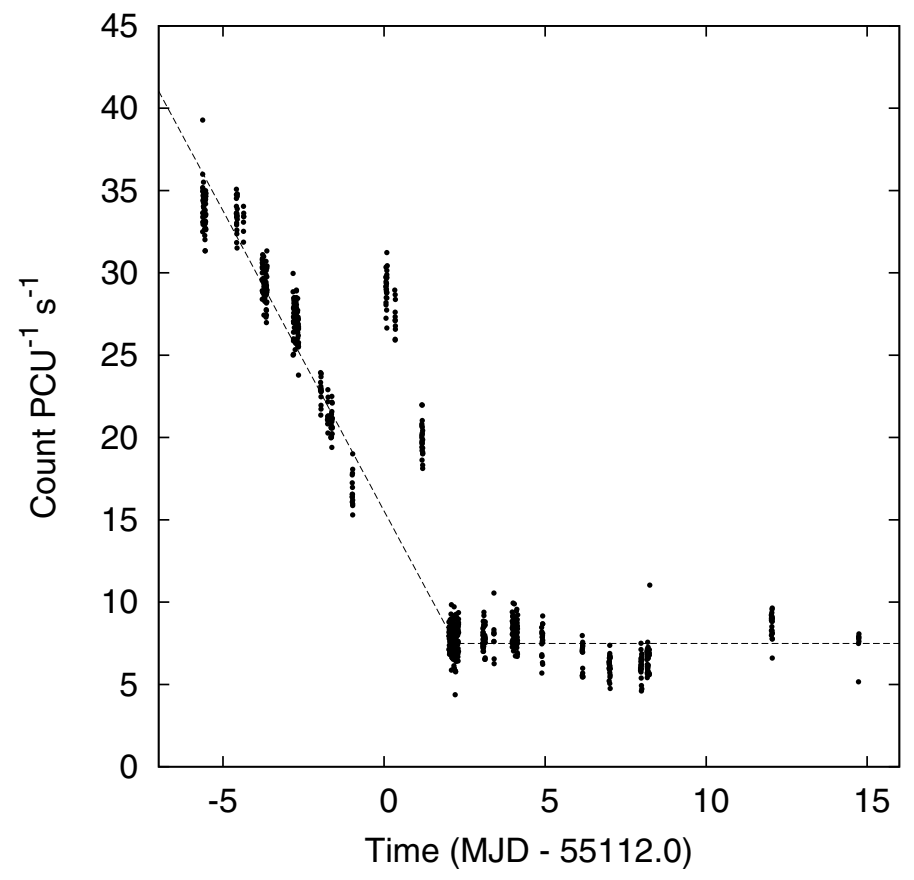

Fig. 1. PCU 2 count rate (2-18 keV), after subtraction of its background, is reported as a function of time in the period from 2 October 2009 and 22 October 2009. During the first few days, the last phase of the IGR J17511-3057 outburst is visible. The flux re-brightening is caused by the onset of the XTE J1751-305 outburst, which lasted less than two days. The remaining days show the constant flux due to the Galactic ridge. The superimposed model is the best-fit using a piecewise linear function. Since we are interested in determine the background due to IGR J17511-3057 and the Galactic ridge, we excluded from the fit the XTE J1751-305 outburst. See the text for more details.

the energy band that maximises the signal-to-noise ratio $(S / N)$ comparing the source X-ray spectrum and the background. We chose the $2-18 \mathrm{keV}$ energy band. We corrected the photon arrival times for the motion of the earth-spacecraft system with respect to the solar system barycentre, and then to barycentric dynamical times at the solar system barycentre using the faxbary tool (DE-405 solar system ephemeris), adopting the Chandra source position reported by $\mathrm{M} 02$. The uncertainty in the source position quoted by M02 is $0.6^{\prime \prime}$, at the $90 \%$ confidence level, as shown in Table 1.

We obtained a first estimate of the mean spin frequency by constructing a Fourier power density spectrum of the $3.2 \mathrm{ks}$ exposure ObsID 94041-01-04-08 data and calculating 53 power spectra from 64-s long data segments $\left(2^{-11}\right.$ bin size), which were averaged into one power spectrum. As reported in Markwardt et al. (2009), we found a signal at $\sim 435.32 \mathrm{~Hz}$. No conclusive evidence of pulsations in the subsequent observations was found in this preliminary step.

\subsection{Determination of the local $T^{\star}$}

We were unable to perform a timing analysis to obtain an orbital solution at the time of the latest outburst from XTE J1751305 because of the weakness of the pulsation. However, we were still able to correct the time series for delays induced by the orbital motion adopting the orbital parameters of the April 2002 outburst estimated by Papitto et al. (2008, P08 hereafter). Propagating the uncertainty in the orbital period $P_{\text {orb }}$ given by P08 across the $\sim 7$ years separating the 2009 outburst from the 2002 one, resulted in an uncertainty in the time of passage through the ascending node, $T^{\star}$, in the 2009 outburst

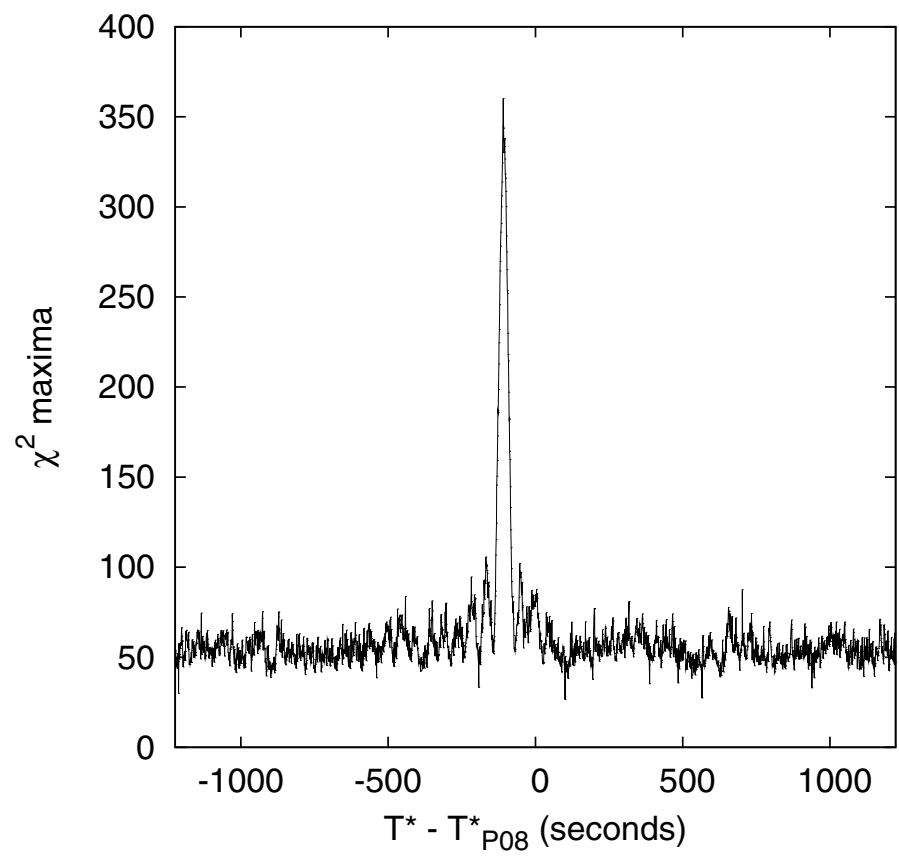

Fig. 2. $\chi^{2}$ maxima obtained from an epoch-folding search on the 2009 data corrected for the orbital modulation, varying each time the epoch of passage through the ascending node with $1 \mathrm{~s}$ step. A total of 2546 orbital solutions were tried, exploring all the possible values for $T^{\star}$.

of $\sim 186 \mathrm{~s}$ (at the $1 \sigma$ confidence level), which is about $\sim 7 \%$ of the orbital period. Moreover, a non-zero orbital period derivative $\dot{P}_{\text {orb }}$ might introduce a further shift in $T^{\star}$. The true local value of $T^{\star}$ can thus differ significantly from the nominal value obtained by propagating the orbital solution provided by P08.

To determine the best local orbital solution, we make the reasonable hypothesis that the best local set of orbital parameters is the one which gives the highest $S / N$, that is, in our case, the highest $\chi^{2}$ value in an epoch-folding search (see e.g. Kirsch et al. 2004). We restrict our search to just one orbital parameter, $T^{\star}$, which is the parameter with the largest uncertainty during the 2009 outburst. To explore all the possible values for $T^{\star}$, the orbital period being $\simeq 2546 \mathrm{~s}$, we produced 2546 different time series from the data of the 2009 outburst, which were corrected for the orbital modulation. For each of these time series, the adopted orbital parameters were the same, except for $T^{\star}$, which is varied in steps of $1 \mathrm{~s}$. We then performed an epoch-folding search for the spin period on each of the 2546 time series using 32 phase bins to sample the signal. In Fig. 2, we show the maximum of the $\chi^{2}$ obtained from the epoch-folding search of each time series as a function of the $T^{\star}$ adopted to produce the time series on which the folding search has been performed.

We fitted the $\chi^{2}$ maxima curve with a model consisting of a constant plus a Gaussian. A clear peak at $\Delta T^{\star} \simeq-110 \mathrm{~s}$ is evident, well within the $1 \sigma$ confidence level of the orbital solution given by $\mathrm{P} 08$ propagated to the 2009 outburst. Thus, we derived the time of passage from the ascending node during the 2009 outburst as $T_{09}^{\star}=T_{\mathrm{P} 08}^{\star}+\Delta T^{\star}$.

Adopting the new value of $T^{\star}$, we barycentered all the data covering the 2009 outburst of XTE J1751-305 and again performed an epoch folding search of the spin period. The improvement in the orbital solution allowed us to detect the pulsation in two additional observations, corresponding to ObsID 9404101-04-04 (MJD 55 112.335, with an exposure of $1.3 \mathrm{ks}$ ) and ObsID 94042-01-02-00 (MJD 55 113.165, with an exposure of $3.1 \mathrm{ks}$, see Table 2 for details). 


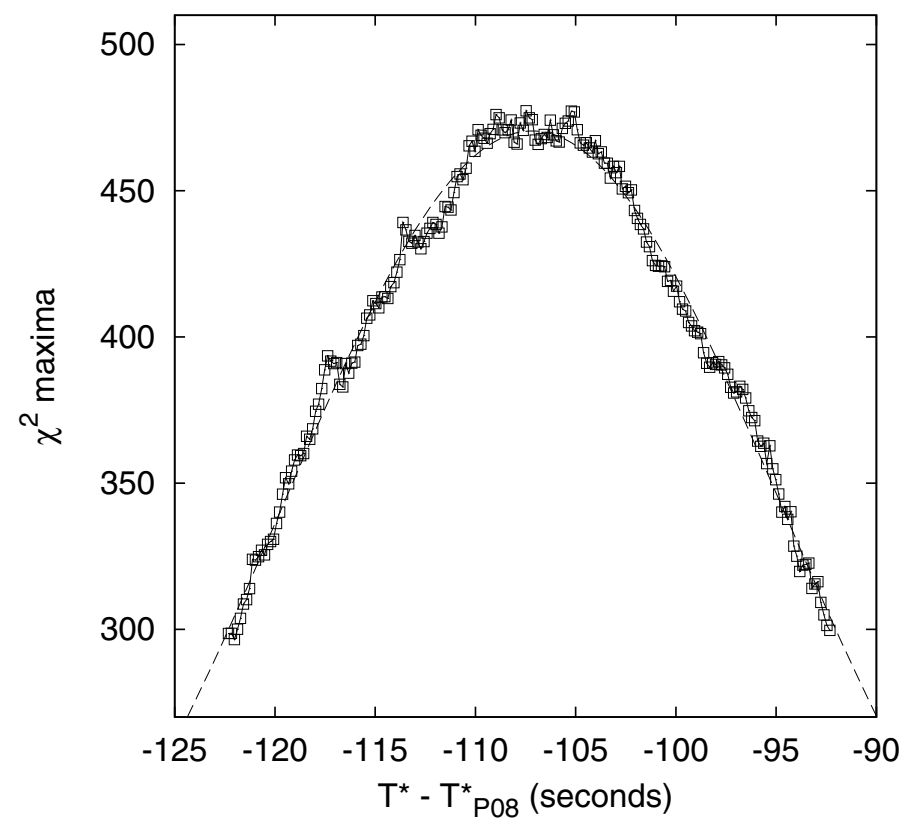

Fig. 3. Maximum of the $\chi^{2}$ obtained in an epoch-folding search around the expected spin period as a function of the $T^{\star}$ adopted to correct the time series for the delays induced by the orbital motion. In this figure we show the result of the final search performed on all the three observations for which the pulsation was detected after having corrected for the orbital motion using the value $T^{\star}$ as estimated from Fig. 2. A $T^{\star}$ step of $0.15 \mathrm{~s}$ was adopted and $200 T^{\star}$ were tried. The best-fit model, constant + Gaussian, is also shown (dashed curve).

We applied the procedure described above considering all the three datasets to refine our new $T_{09}^{\star}$ measurement. We adopted a finer step in $T^{\star}$ of $0.15 \mathrm{~s}$, covering an interval of $30 \mathrm{~s}$ around the new value of $T^{\star}$. We again fitted the $\chi^{2}$ maxima versus (vs.) $T^{\star}$ curve with a model consisting of a constant plus a Gaussian, as shown in Fig. 3. In this way, we were able to obtain a precise measurement of $T^{\star}$; the final value for $T^{\star}$ is reported in Table 1 . The evaluation of the uncertainty in $T^{\star}$ is discussed in the next section.

\subsection{Error estimates in $T^{\star}$ using Monte Carlo simulations}

The folding search technique described above and adopted to obtain our refined measure of $T^{\star}$ does not provide a straightforward determination of the uncertainty in this parameter. To overcome this problem, we performed a Monte Carlo simulation. We generated 100 datasets with the same exposure, count rate, pulse modulation, and orbital modulation observed in the real data. For each of these dataset, we applied the same procedure as described in the previous section to obtain a measure of $T^{\star}$. The confidence level at $68 \%(1 \sigma)$ for the $T^{\star}$ parameter corresponds to $1.05 \mathrm{~s}$, which is about 30 times the corresponding confidence interval obtained by a fit with a Gaussian of the $\chi^{2}$ maxima vs. $T^{\star}$ curve. We therefore adopt $1.05 \mathrm{~s}$ as our best estimate of the $1 \sigma$ uncertainty on the $T^{\star}$ measure.

\subsection{Timing analysis}

We barycentered our data of XTE J1751-305 using our refined orbital solution, and performed an epoch-folding search to determine a mean spin frequency for the 2009 outburst. From the best-fit of the $\chi^{2}$ curve, we obtained a value of 435.31799237(19) Hz. We evaluated the frequency uncertainty
Table 1. Orbital and spin parameters for XTE J1751-305.

\begin{tabular}{|c|c|c|}
\hline Parameter & Value & \\
\hline RA (J2000) & $17^{\mathrm{h}} 51^{\mathrm{m}} 13.49(5)$ & $a$ \\
\hline $\operatorname{Dec}(\mathrm{J} 2000)$ & $-30^{\circ} 37^{\prime} 23^{\prime \prime} .4(6)$ & $a$ \\
\hline Projected semi-major axis $a_{x} \sin i(\mathrm{lt}-\mathrm{ms})$ & $10.125(5)$ & $b$ \\
\hline \multicolumn{3}{|l|}{ Ascending node passage, $T^{\star}$ (MJD) at } \\
\hline 2002 outburst & $52368.0129023(4)$ & $b$ \\
\hline 2009 outburst & $55111.000647(12)$ & \\
\hline Orbital period, $P_{\text {orb }}(\mathrm{s})$ & $2545.342(2)$ & $b$ \\
\hline Eccentricity, $e$ & $<1.3 \times 10^{-3}$ & $b$ \\
\hline Reference epoch, $T_{0}$ (MJD) & 55112.0 & \\
\hline Mean spin frequency, $v_{0}(\mathrm{~Hz})$ & $435.31799256(23)$ & \\
\hline $\begin{array}{l}\text { Secular spin frequency derivative, } \\
\qquad \dot{v}_{\mathrm{S}}\left(\mathrm{Hz} \mathrm{s}^{-1}\right)\end{array}$ & $-0.55(12) \times 10^{-14}$ & \\
\hline
\end{tabular}

Notes. Errors are at $1 \sigma$ confidence level, upper limits are given at $95 \%$ confidence level. Errors in the source position are given at $90 \%$ confidence level. Times are referred to the barycentre of the Solar System (TDB).

References. ${ }^{a}$ Markwardt et al. (2002); ${ }^{b}$ Papitto et al. (2008).

Table 2. Exposures and fractional amplitudes of the analysed Obs Ids.

\begin{tabular}{lccc}
\hline \hline Obs ID & $\begin{array}{c}\text { Start time } \\
(\mathrm{MJD})\end{array}$ & $\begin{array}{c}\text { Exposure } \\
(\mathrm{ks})\end{array}$ & $\begin{array}{c}\text { Fractional amplitude } \\
(\%)\end{array}$ \\
\hline $94041-01-04-08$ & 55112.052 & 3.2 & $7.9 \pm 1.0$ \\
$94041-01-04-04$ & 55112.335 & 1.3 & $7.6 \pm 2.2$ \\
$94042-01-02-00$ & 55113.165 & 3.1 & $7.1 \pm 2.0$ \\
\hline
\end{tabular}

Notes. For each Obs Id in which pulsations at the frequency of XTE J1751-305 have been detected in this work, the start time, the exposure, and the fractional amplitude (corrected for the contribution of IGR J17511-3057, Galactic ridge, and instrumental background) are reported.

using the Monte Carlo simulated data described above. We found that the error determined in this way was an order of magnitude larger than the error determined by fitting with a Gaussian the centroid position of the epoch-folding search curve.

Adopting this mean spin frequency value, we epoch-folded the three observations during which pulsations could be detected over $1000 \mathrm{~s}$ long intervals, considering 16 bins to sample the signal (see Table 2 for details). In this way, we obtained seven folded pulse profiles. We performed an harmonic decomposition of each pulse profile. The fundamental and the first overtone were significantly detected. The fundamental was significantly detected in six folded pulse profiles, while the first overtone only in one profile. We fitted the pulse phase delays with a constant plus a linear term, representing a constant (mean) spin frequency model. From the fit, we obtained a mean spin frequency of $435.31799256(22)$, with a final $\chi^{2} /$ d.o.f. of 1.55 (4 d.o.f.). This value is in perfect agreement with the value obtained with the epoch-folding search. We note that the uncertainty in the pulse frequency obtained from the fitting of the pulse phase delays fit is nearly equal to the uncertainty in the pulse frequency obtained with a folding search estimated with the Monte Carlo simulations, so that both procedures give consistent results. The pulse phase delays and the best-fit line are shown in Fig. 4. The pulse profile obtained by folding all the data is shown in Fig. 5.

To correctly determine the fractional amplitude, an estimate of the background and the contribution from sources contaminating the field of view is mandatory. In this case, the major contribution to the background is due to the emission of 


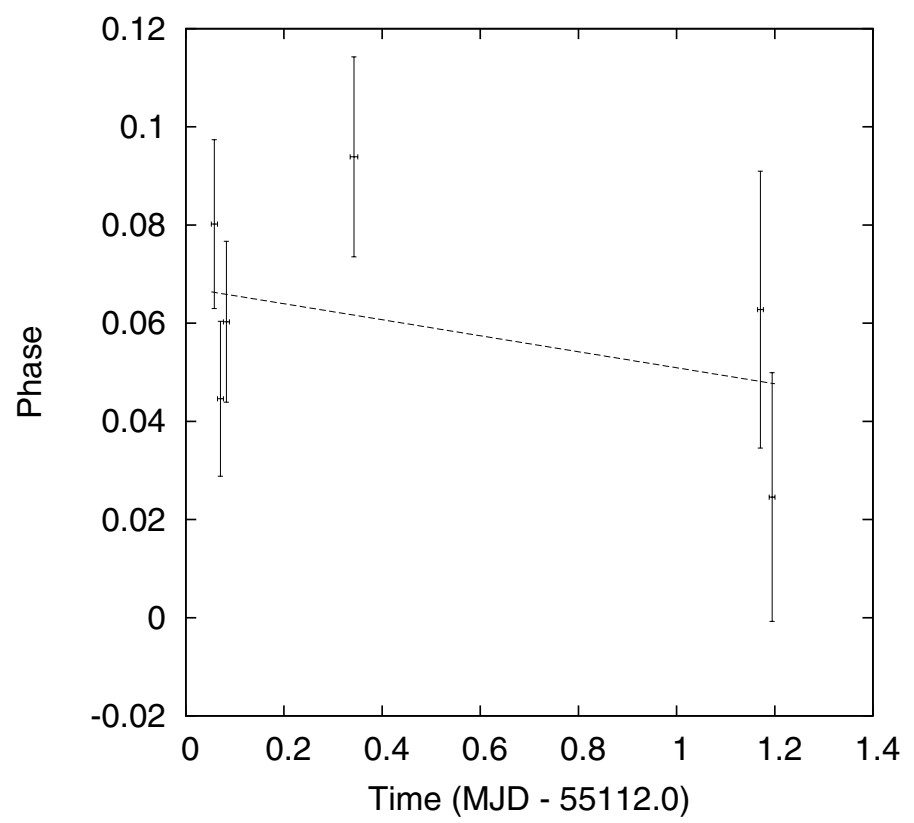

Fig. 4. Pulse phase delays of the fundamental for the three observations in which the pulsation is detected. Each phase point is obtained folding on $\sim 1000$-s long time intervals and using a spin frequency $v=435.31799237$, which is the value obtained with the epoch-folding search technique. The best-fit constant spin frequency model is also shown (dashed line).

the AMP IGR J17511-3057 and of the Galactic ridge. While the roughly constant contribution from the Galactic ridge is $\sim 7.5 \mathrm{cts} \mathrm{s}^{-1}$ in the considered energy band (Papitto et al. 2010), the IGR J17511-3057 contribution can only be estimated extrapolating its flux decay trend just before the XTE J1751305 outburst onset. We fitted the 2-18 keV IGR J17511-3057 $\mathrm{X}$-ray light curve with a linear model in the time interval from 55108.92 to 55111.46 MJD, as can be seen in Fig. 1. In Table 2, we report the fractional amplitudes obtained for each of the three observation. These values are corrected with respect to the instrumental background, as well as the estimated contribution from IGR J17511-3057 and the Galactic ridge, but are still strongly dependent on the model adopted to describe the IGR J17511-3057 X-ray light curve.

The uncertainty in the position of the source quoted by M02 is 0.6" (90\% confidence level, see Aldcroft et al. 2000), while $0.37^{\prime \prime}$ is the confidence interval corresponding to $1 \sigma^{1}$. Because of this, a systematic uncertainty arises on the spin frequency obtained by fitting the pulse phase delays. These systematic uncertainties are $\sigma_{v \text { sys }}=4.8 \times 10^{-7} v_{3} \sigma^{\prime \prime}\left(1+\sin ^{2} \beta\right)^{1 / 2} \mathrm{~Hz}$ (Burderi et al. 2007), where $v_{3}$ is the spin frequency in units of $1000 \mathrm{~Hz}, \sigma^{\prime \prime}$ is the positional error circle in units of arcsec, and $\beta$ refers to the ecliptic latitude of the source (for XTE J1751-305 $\lambda=268.097281^{\circ}$ and $\beta=-7.198364^{\circ}$ ). With these values, we have $\sigma_{v \text { sys }}=7.8 \times 10^{-8} \mathrm{~Hz}$.

Combining in quadrature this systematic error with the statistical error of $2.2 \times 10^{-7} \mathrm{~Hz}$, we find a final error in the spin frequency of $\sigma_{v}=2.3 \times 10^{-7} \mathrm{~Hz}$. Thus, the value of the average spin frequency during the 2009 outburst is $\bar{v}_{09}=$ 435.31799256(23) Hz.

\footnotetext{
${ }^{1}$ See http://cxc.harvard.edu/cal/ASPECT/celmon/
}

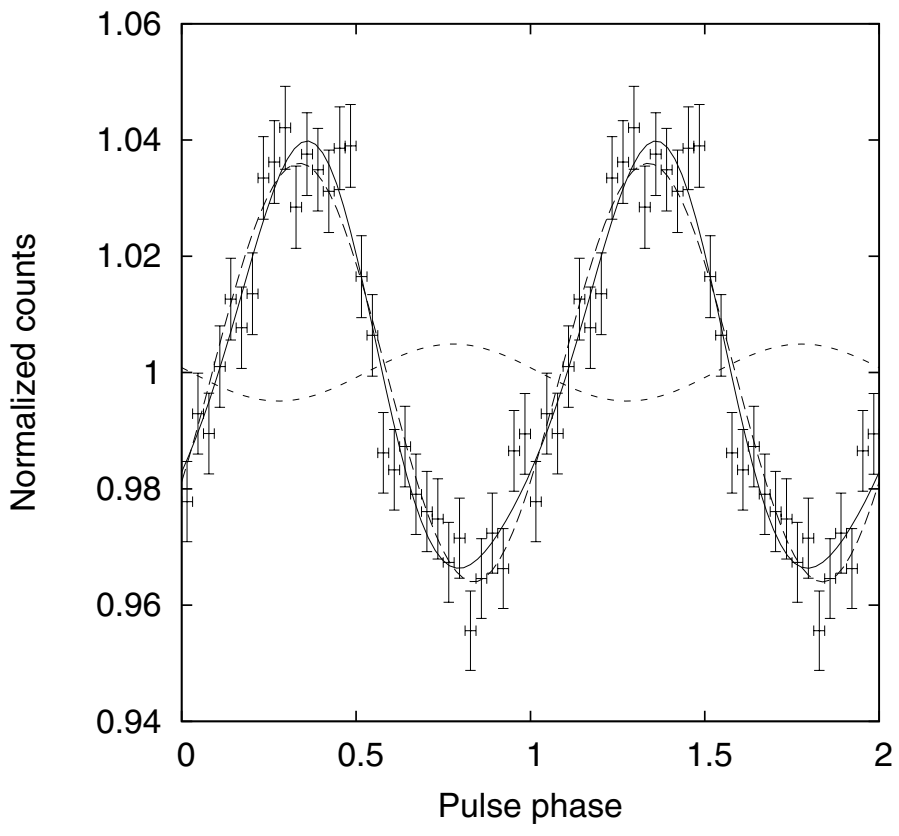

Fig. 5. Folded pulse profile of the three datasets in which the pulsation was detected. The profile is reported twice for clarity.

\section{Discussion}

We have obtained a refined orbital solution and a precise estimate of the spin frequency of the AMP XTE J1751-305 from a timing analysis of the RXTE data during its 2009 outburst.

\subsection{Orbital period evolution}

Although a measure of $\dot{P}_{\text {orb }}$ is impossible with only two measurements of $T^{\star}$, we can derive an upper and lower limit to the $\dot{P}_{\text {orb }}$ using the full orbital solution given by P08 for the 2002 outburst and our measure of $T^{\star}$ for the 2009 outburst. Using Eq. (1) given in Burderi et al. (2009), we derive $\dot{P}_{\text {orb }}$ to be

$\dot{P}_{\text {orb }}=\frac{2}{N P_{\text {orb }}}\left(\frac{\Delta T^{\star}(N)}{N}-\Delta P_{\text {orb }}\right)$,

where $P_{\text {orb }}$ is the orbital period measured by P08, $N(=93109)$ is the integer number of orbital cycles between the two $T^{\star}$, and $\Delta T^{\star}(N)$ is the difference between the measured $T^{\star}$ at the $N$ th orbital cycle and its expected value, that is $\Delta T^{\star}(N)=T_{09}^{\star}$ $\left(T_{P 08}^{\star}+N \times P_{\text {orb }}\right)$. We can assume that the correction term in Eq. (1), $\Delta P_{\text {orb }}$, is at most the confidence interval for $P_{\text {orb }}$ given by P08. Considering the maximum and minimum values of $P_{\text {orb }}$ within its confidence interval, we find $\dot{P}_{\text {orb }}$ to be in the interval from $-2.7 \times 10^{-11}$ to $+0.7 \times 10^{-11} \mathrm{~s} \mathrm{~s}^{-1}$, at $1 \sigma$ confidence level.

\subsection{Spin frequency secular evolution}

We derive the secular spin frequency derivative comparing our measurement of the spin frequency for the 2009 outburst with the spin frequency measured by P08 analysing the XTE J17513052002 outburst. Moreover, we consider the possible effects on the spin frequency of the two outbursts that occurred in the intervening seven years. To compute the effect on the spin frequency of the two weak outbursts between the 2002 and the 2009 outbursts, we assume that, during each outburst, the neutron star (NS) is accreting angular momentum $L$ at a rate

$\mathrm{d} L / \mathrm{d} t=\dot{M} \sqrt{G M R_{\mathrm{a}}}$, 
Table 3. Spin frequency values for all the observed XTE J1751-305 outbursts.

\begin{tabular}{|c|c|c|c|c|c|c|c|}
\hline Outburst & $\begin{array}{c}v_{\mathrm{i}} \\
(\mathrm{Hz})\end{array}$ & $\begin{array}{c}v_{\mathrm{f}} \\
(\mathrm{Hz})\end{array}$ & $\begin{array}{c}\bar{v} \\
(\mathrm{~Hz})\end{array}$ & $\begin{array}{c}F_{\mathrm{X} \mathrm{i}} \\
\left(10^{-11} \mathrm{erg} \mathrm{cm}^{-2} \mathrm{~s}^{-1}\right)\end{array}$ & $\begin{array}{c}\tau \\
\text { (days) }\end{array}$ & $\begin{array}{c}\Delta v \\
\left(10^{-8} \mathrm{~Hz}\right)\end{array}$ & References \\
\hline 2002 & $435.31799357(9)$ & $435.31799385(16)$ & - & $134(7)$ & $7.1(1)$ & $28(8)$ & $a$ \\
\hline 2005 & - & - & - & $19(7)$ & $\simeq 2.4$ & $1.5(4)$ & $b$ \\
\hline 2007 & - & - & - & $24(5)$ & $\simeq 2.3$ & $1.7(3)$ & $c$ \\
\hline 2009 & $435.31799255(23)$ & - & $435.31799256(23)$ & $36(14)$ & $2.4(2)$ & $0.8(3)-1.7(6)$ & $d$ \\
\hline
\end{tabular}

Notes. We report, for each outburst, the spin frequency at the start of the outburst $\left(v_{\mathrm{i}}\right)$, the spin frequency at the end of the outburst $\left(v_{\mathrm{f}}\right)$, the average spin frequency $(\bar{v})$, the flux at the peak of the outburst in the energy range $2-10 \mathrm{keV}\left(F_{\mathrm{Xi}}\right)$, the flux decay time scale $\tau$ in the hypothesis of an exponential decay, and the inferred spin frequency variation during the outburst.

References. ${ }^{a}$ Markwardt et al. (2002), P08; ${ }^{b}$ Grebenev et al. (2005); Swank et al. (2005); ${ }^{c}$ Falanga et al. (2007); Markwardt \& Swank (2007); ${ }^{d}$ this work, Chenevez et al. (2009).

where $\dot{M}$ is the mass accretion rate, $\mathrm{G}$ is the gravitational constant, $M$ is the NS mass, and $R_{\mathrm{a}}$ is the radius at which the accreting matter (orbiting with Keplerian speed in an accretion disc) is quickly removed from the disc by the interaction with the NS magnetic field. We assume the working hypothesis that the magnetospheric radius $R_{\mathrm{a}}$ can be expressed as (see Rappaport et al. 2004, and references, therein)

$R_{\mathrm{a}} \propto \dot{M}^{-\frac{2}{7}}$,

where $\dot{M}$ is the mass accretion rate, and that the mass accretion rate is proportional to the X-ray flux $F_{\mathrm{X}}$. As reported by Markwardt et al. (2002), the light-curve of XTE J1751-305 during the 2002 outburst showed an exponential decay followed by a sharp break after which the flux quickly dropped below detectability. We therefore decided to model the X-ray flux $F_{\mathrm{X}}$ of each outburst displayed by the source with the function

$F_{\mathrm{X}}= \begin{cases}F_{\mathrm{Xi}} \exp (-t / \tau) & \text { if } 0 \leq t \leq \bar{t} \\ 0 & \text { if } t<0 \text { or } t>\bar{t},\end{cases}$

where $F_{\mathrm{X}}$ is the X-ray flux at the outburst peak, $\bar{t}$ is the duration of the exponential decay, and $\tau$ the decay time. In Table $3, F_{\mathrm{X}}$ i and $\tau$ are reported for each outburst. Since the 2002 outburst is the only one for which a measure of the spin frequency derivative was possible, we use it as a reference for the other outbursts. Using the above equations and hypotheses, we can derive the spin frequency derivative and, integrating over time, the spin frequency variation $\Delta v$ in an outburst of time-length $\bar{t}$. After some algebraic manipulation, we find that

$\Delta v=\dot{v}_{\mathrm{i} 02} \tau^{*}\left[1-\exp \left\{-\frac{\bar{t}}{\tau^{*}}\right\}\right]\left(\frac{F_{\mathrm{Xi}}}{F_{\mathrm{X} \mathrm{i} 02}}\right)^{\frac{6}{7}}$,

where $\tau^{*}=7 \tau / 6, F_{\mathrm{Xi} 02}$ is the X-ray flux at the beginning of the 2002 outburst and $\dot{v}_{\mathrm{i} 02}$ is the corresponding spin frequency derivative. The flux and the spin frequency derivative of the 2002 outburst are $F_{\mathrm{Xi} 02}=1.34(7) \times 10^{-9} \mathrm{erg} \mathrm{cm}^{-2} \mathrm{~s}^{-1}(2-10 \mathrm{keV})$, and $\dot{v}_{\mathrm{i} 02}=0.56(12) \times 10^{-12} \mathrm{~Hz} \mathrm{~s}^{-1}$ (see P08).

For the same outburst, M02 obtained parameters of $\tau=$ 7.1(1) days and $\bar{t}=8.5$ days, whose ratio is $\tau / \bar{t} \simeq 0.84$. Adopting the same model for the 2009 outburst, we found that $\tau=2.4(2)$ days and $\bar{t}$ is in the range 1.2-2.9 days, which implies that the ratio $\tau / \bar{t}$ is in the range $0.83-2.0$.

\subsubsection{Spin-down between 2002 and 2009 outbursts}

Because a significant spin-frequency derivative was detected during the 2002 outburst (P08), we considered two frequencies, at the beginning and at the end of the 2002 outburst, respectively. The frequency at the beginning of the 2002 outburst, at $T_{0}=52368.653 \mathrm{MJD}$, is $v_{02} \mathrm{i}=435.31799357(9) \mathrm{Hz}$. As usual, the error is on the last digit at $1 \sigma$ level and was computed by combining in quadrature the statistical error in the spin frequency estimate, $4 \times 10^{-8} \mathrm{~Hz}$, with the systematic error induced by the uncertainty in the source position, $7.8 \times 10^{-8} \mathrm{~Hz}$. The frequency at the end of the outburst, which occurred about nine days after the beginning, when the pulsation was detected for the last time, is $v_{\mathrm{f} 02}=435.31799385(16)$, which was computed adopting the mean value for the spin frequency derivative given in $\mathrm{P} 08, \dot{v}=3.7(1.0) \times 10^{-13} \mathrm{~Hz} \mathrm{~s}^{-1}$. The error is again computed by combining in quadrature the statistical and systematic uncertainties in $v_{0}\left(4 \times 10^{-8} \mathrm{~Hz}\right.$ and $7.8 \times 10^{-8} \mathrm{~Hz}$, respectively $)$ with the statistical and systematic uncertainties in the spin frequency derivative $\left(7.8 \times 10^{-8} \mathrm{~Hz}\right.$ and $1.2 \times 10^{-8} \mathrm{~Hz}$, respectively), where the systematic error in the spin frequency derivative induced by the uncertainty in the source position (Burderi et al. 2007) is $\sigma_{\dot{v} \text { sys }}=9.6 \times 10^{-14} v_{3} \sigma^{\prime \prime}\left(1+\sin ^{2} \beta\right)^{1 / 2} \mathrm{~Hz} \mathrm{~s}^{-1}=1.6 \times$ $10^{-14} \mathrm{~Hz} \mathrm{~s}^{-1}$ for XTE J1751-305. For the 2009 outburst, we considered that $v_{09} \simeq \bar{v}_{09}$. Averaging Eq. (5) over the outburst length $\bar{t}$, we obtain an expression for the spin frequency at the beginning of the 2009 outburst

$v_{\mathrm{i} 09}=\bar{v}_{09}-\dot{v}_{\mathrm{i} 02} \tau^{*}\left\{1-\frac{\tau^{*}}{\bar{t}}\left[1-\exp \left\{-\frac{\bar{t}}{\tau^{*}}\right\}\right]\right\}\left(\frac{F_{\mathrm{X} \mathrm{i} 09}}{F_{\mathrm{X} \mathrm{i} 02}}\right)^{\frac{6}{7}}$.

The 2009 outburst length lies in the range 1.2-2.9 days, where 1.2 days is the time interval in which the pulsation was detected, while 2.9 days is the maximum possible length of the outburst (see Fig. 1). Assuming $F_{\mathrm{X} \text { i } 09}=36 \times 10^{-11} \mathrm{erg} \mathrm{cm}^{-2} \mathrm{~s}^{-1}$, $\tau=2.4$ (2) days, and $\bar{t}=1.2$, Eq. (6) gives $v_{\mathrm{i} 09}=\bar{v}_{09}-$ $0.8(3) \times 10^{-8} \mathrm{~Hz}=435.31799255(23) \mathrm{Hz}$, while considering $\bar{t}=$ 2.9 gives $v_{\mathrm{i} 09}=\bar{v}_{09}-1.7(6) \times 10^{-8} \mathrm{~Hz}=435.31799254(23) \mathrm{Hz}$. The difference between the two cases is, for our purposes, irrelevant.

To compute the secular spin frequency derivative, we consider the total time elapsed from the end of the 2002 outburst to the beginning of the 2009 outburst, namely $\Delta t=$ 55 112.0-52 377.653 MJD. The secular spin frequency derivative is

$\dot{v}_{\mathrm{s}}=\frac{\left(v_{\mathrm{i} 09}-v_{\mathrm{f} 02}\right)}{\Delta t}=-0.55(12) \times 10^{-14} \mathrm{~Hz} \mathrm{~s}^{-1}$,

where the $1 \sigma$ error is computed by adding in quadrature all the errors. We note that this value is about one order of magnitude higher than the secular spin-down rate measured for the AMP SAX J1808.4-3658 (Hartman et al. 2009), and of the same order of magnitude as the secular spin-down rate measured for 
the fastest AMP IGR J00291+5934 (Patruno 2010; Hartman et al. 2011; Papitto et al. 2011). Even in the worst case scenario, that is if we computed the secular spin frequency derivative neglecting the frequency variation in all the 2002 outburst (in this case, the total time elapsed has been computed as $\Delta t=55112.0$ $52368.653 \mathrm{MJD}$, namely the total time elapsed from the beginning of the 2002 outburst to the beginning of the 2009 outburst), we obtain $\dot{v}_{\mathrm{s}}=-0.43(10) \times 10^{-14} \mathrm{~Hz} \mathrm{~s}^{-1}$, which is still significant and compatible, within the errors, with the previous value.

\subsubsection{Effect of the spin-up during 2005 and 2007 outbursts}

We now consider that on 28 March 2005 and 4 April 2007, two additional outbursts occurred. In the following, we discuss the effect of a possible spin-up during these two outburst, similar to the one observed for the 2002 outburst. It should be noted here that there is a possibility that the 2005 outburst is not associated with XTE J1751-305, since no precise position for the source of the outburst is available for the 2005 event. Using Eq. (5), we can evaluate an order of magnitude estimate of the spin frequency variation that may have occurred during these two outbursts.

For the second outburst, which occurred on 28-29 March 2005 , the peak flux was $19(7) \times 10^{-11} \mathrm{erg} \mathrm{cm}^{-2} \mathrm{~s}^{-1}(2-10 \mathrm{keV})$, and $\bar{t} \simeq 2.4$ days (Grebenev et al. 2005; Swank et al. 2005).

For the third outburst, on 4-5 April 2007, the peak flux was $24(5) \times 10^{-11} \mathrm{erg} \mathrm{cm}^{-2} \mathrm{~s}^{-1}(2-10 \mathrm{keV})$, and $\bar{t} \simeq 2.3$ days (Falanga et al. 2007; Markwardt \& Swank 2007). With these values, using Eq. (5) and adopting $\bar{t} / \tau \simeq 1$, we derive $\Delta v_{05}=1.5(4) \times 10^{-8} \mathrm{~Hz}$ and $\Delta v_{07}=1.7(3) \times 10^{-8} \mathrm{~Hz}$, where the errors were computed by propagating the uncertainties in the fluxes. The above results are obtained in the hypothesis that $\bar{t} / \tau \simeq 1$. The result holds even if we consider, as in the case of the 2009 outburst, that $\tau=2 \bar{t}$. In this case the frequency changes in the two outbursts are $\Delta v_{05}=1.8(5) \times 10^{-8} \mathrm{~Hz}$ and $\Delta v_{07}=2.1(4) \times 10^{-8} \mathrm{~Hz}$. It is clear that the effect on the value of $\dot{v}_{\mathrm{s}}$, considering only the 2007 outburst or considering both the 2005 and 2007 outbursts, is negligible. We have thus demonstrated that, within the error, the secular spin frequency derivative is independent of the frequency variations caused by possible spin-up episodes during the weak 2005 and 2007 outbursts.

\subsection{Magnetic field}

Using the derived secular spin frequency derivative, we can estimate the magnetic field strength by equating the rotationalenergy loss rate to the rotating magnetic dipole emission. It is unclear which expression should be used to evaluate the energy radiated by a rotating dipole. While the classical formula for a rotating dipole in a vacuum is well known, an equivalent expression in the presence of matter has yet to be derived. Goldreich \& Julian (1969) demonstrated that NS typical magnetic field strengths are strong enough to fill the magnetosphere with charged particles extracted from the surface, with the result that even an aligned rotator emits energy. We can write the amount of energy radiated as (Spitkovsky 2006)

$\dot{E} \simeq-f(\theta) \mu^{2} c^{-3} \omega^{4}$,

where $c$ is the speed of light, $\mu$ is the magnetic dipole moment, $\omega$ the NS angular spin frequency, $\theta$ the angle between the rotation and magnetic axes, and $f(\theta)$ a dimensionless function that takes into account the energy dependence of angle $\theta$ and the effects of the presence of particles in the magnetosphere. In a vacuum, $f(\theta)=2 / 3 \sin ^{2}(\theta)$, while in the case of matter in the magnetosphere, Spitkovsky (2006) proposed, on the basis of MHD simulations, that $f(\theta)=1+\sin ^{2}(\theta)$ (see Contopoulos 2007, for more details). Equating the irradiated energy to the rotational energy loss rate, we obtain

$\mu=\sqrt{\frac{I c^{3} \dot{\omega}}{f(\theta) \omega^{3}}} \simeq 8.27 \times 10^{26} f^{-\frac{1}{2}}(\theta) I_{45}^{\frac{1}{2}} v_{2}^{-\frac{3}{2}} \dot{v}_{-15}^{\frac{1}{2}} \mathrm{G} \mathrm{cm}^{3}$

where $I_{45}$ is the NS moment of inertia in units of $10^{45} \mathrm{~g} \mathrm{~cm}^{2}$, $v_{2}$ the spin frequency in units of $100 \mathrm{~Hz}$, and $\dot{v}_{-15}$ the spin frequency derivative in units of $10^{-15} \mathrm{~Hz} \mathrm{~s}^{-1}$. Using our estimates of the spin frequency and its secular derivative reported in Table 1, we obtain a value for the magnetic dipole strength of $\mu=2.14(23) \times 10^{26} f^{-\frac{1}{2}}(\theta) I_{45}^{1 / 2} \mathrm{G} \mathrm{cm}^{3}$.

Assuming a $1.4 M_{\odot}$ NS mass and adopting the FPS (see Friedman \& Pandharipande 1981; Pandharipande \& Ravenhall 1989) equation of state, we obtain a radius of $R_{\mathrm{NS}}=1.14 \times 10^{6} \mathrm{~cm}$ and a moment of inertia $I=1.29 \times 10^{45} \mathrm{~g} \mathrm{~cm}^{2}$. Under the assumptions above, we estimate the magnetic field strength at the magnetic caps $B_{\mathrm{PC}}$ from the relation that gives the dipole magnetic field strength at the NS surface as a function of the magnetic dipole moment and the angle $\alpha$ between the position on the surface and the magnetic dipole axis $(\alpha=0$ on the magnetic cap) $B=\left(\mu / R_{\mathrm{NS}}^{3}\right) \sqrt{1+3 \cos ^{2} \alpha}$. We find $B_{\mathrm{PC}}=$ $3.3(4) \times 10^{8} f^{-\frac{1}{2}}(\theta)$ G. Adopting $f(\theta)=2 / 3$ (in line with what is assumed when deriving the magnetic field of radio pulsars), we find $B_{\mathrm{PC}}=4.0(4) \times 10^{8} \mathrm{G}$, which is quite reasonable for this kind of source.

Hartman et al. (2011) noted that the AMPs for which the secular spin-down was measured are suitable to be detected as $\gamma$-ray millisecond pulsars by the Fermi Large Area Telescope, since several millisecond radio pulsars with similar characteristics were detected (see Abdo et al. 2009). The spin-down power, defined as $\dot{E}=4 \pi^{2} I \omega \dot{\omega}$, for this source is $\dot{E}=0.9(2) \times$ $10^{35} I_{45} \mathrm{erg} \mathrm{s}^{-1}$. Following Abdo et al. (2009), the upper limit to the $\gamma$-ray flux is $\eta \dot{E} / d^{2} \leq 2.4 \times 10^{33} I_{45} \mathrm{erg} \mathrm{s}^{-1} \mathrm{kpc}^{-2}$, where $\eta$ is the $\gamma$-ray efficiency (see Abdo et al. 2009, for $\eta$ definition) and $d$ is the XTE J1751-305 distance lower limit, estimated by P08 to be $6.3 \mathrm{kpc}$. The observed values for $\eta$ is in the range $6-100 \%$ (Abdo et al. 2009). The chance of detecting XTE J1751-305 in $\gamma$-rays is then unlikely, although possible if the $\gamma$-ray efficiency is high.

For completeness, NS magneto-dipole radiation is not the only means of invoking NS angular momentum loss. The NS mass distribution can deviate from a perfectly spherical distribution for several reasons (see, e.g. Bildsten 1998), introducing a neutron star's mass quadrupole moment that permits the emission of gravitational waves $(\mathrm{GW})$ at a frequency that is double the NS spin frequency. Following Hartman et al. (2011) and Papitto et al. (2011), it is possible to give an upper limit to the average neutron star's mass quadrupole moment $Q$, under the hypothesis that the spin down is due only to GW emission. Using the expression for the net torque due to a mass quadrupole moment given in Thorne (1980) and adopting the value of the spin frequency and its derivative obtained in this work, we can derive an upper limit to the quadrupole ellipticity (see, e.g. Ferrari 2010) $Q / I \leq 4.6 \times 10^{-9} I_{45}^{-1 / 2}$ ( $3 \sigma$ confidence level), in line with the values obtained for the sources SAX J1808.4-3658 (Hartman et al. 2008) and IGR J00291+5934 (Hartman et al. 2011; Papitto et al. 2011). This upper limit also agrees with those obtained for the millisecond radio pulsars. With these values of ellipticity and source distance, the predicted GW amplitude is well below 
the detection threshold of the current GW detectors (e.g. Abbott et al. 2010).

Acknowledgements. We thank A. Possenti for fruitful discussions and the unknown referee for useful suggestions. This work is supported by the Italian Space Agency, ASI-INAF I/088/06/0 contract for High Energy Astrophysics, as well as by the operating program of Regione Sardegna (European Social Fund 20072013), L.R.7/2007, "Promotion of scientific research and technological innovation in Sardinia".

\section{References}

Abbott, B. P., Abbott, R., Acernese, F., et al. 2010, ApJ, 713, 671

Abdo, A. A., Ackermann, M., Ajello, M., et al. 2009, Science, 325, 848

Aldcroft, T. L., Karovska, M., Cresitello-Dittmar, M. L., Cameron, R. A., \&

Markevitch, M. L. 2000, SPIE Conf. Ser. 4012, ed. J. E. Truemper, \&

B. Aschenbach, 650

Altamirano, D., Patruno, A., Heinke, C. O., et al. 2010, The Astronomer's Telegram, 2500, 1

Bildsten, L. 1998, ApJ, 501, L89

Burderi, L., Di Salvo, T., Lavagetto, G., et al. 2007, ApJ, 657, 961

Burderi, L., Riggio, A., di Salvo, T., et al. 2009, A\&A, 496, L17

Chenevez, J., Kuulkers, E., Beckmann, V., et al. 2009, The Astronomer's Telegram, 2235, 1

Contopoulos, I. 2007, in WE-Heraeus Seminar on Neutron Stars and Pulsars 40 years after the discovery, ed. W. Becker, \& H. H. Huang, MPE-Rep., 291 (Garching bei München, Germany: Max Planck Institut für extraterrestrische Physik), Proc., 363, 134

di Salvo, T., Burderi, L., Riggio, A., Papitto, A., \& Menna, M. T. 2008, MNRAS, 389,1851

Falanga, M., Soldi, S., Shaw, S., et al. 2007, The Astronomer's Telegram, 1046, 1

Ferrari, V. 2010, Class. Quant. Grav., 27, 194006
Friedman, B., \& Pandharipande, V. R. 1981, Nucl. Phys. A, 361, 502

Galloway, D. K., Markwardt, C. B., Morgan, E. H., Chakrabarty, D., \& Strohmayer, T. E. 2005, ApJ, 622, L45

Galloway, D. K., Hartman, J. M., Chakrabarty, D., Morgan, E. H., \& Swank, J. H. 2008, The Astronomer's Telegram, 1786, 1

Goldreich, P., \& Julian, W. H. 1969, ApJ, 157, 869

Grebenev, S. A., Molkov, S. V., \& Sunyaev, R. A. 2005, The Astronomer's Telegram, 446, 1

Hartman, J. M., Patruno, A., Chakrabarty, D., et al. 2008, ApJ, 675, 1468

Hartman, J. M., Patruno, A., Chakrabarty, D., et al. 2009, ApJ, 702, 1673

Hartman, J. M., Galloway, D. K., \& Chakrabarty, D. 2011, ApJ, 726, 26

Jahoda, K., Markwardt, C. B., Radeva, Y., et al. 2006, ApJS, 163, 401

Kirsch, M. G. F., Mukerjee, K., Breitfellner, M. G., et al. 2004, A\&A, 423, L9

Markwardt, C. B., \& Swank, J. H. 2007, The Astronomer's Telegram, 1045, 1

Markwardt, C. B., Swank, J. H., Strohmayer, T. E., in 't Zand, J. J. M., \& Marshall, F. E. 2002, ApJ, 575, L21

Markwardt, C. B., Pereira, D., \& Swank, J. H. 2007, The Astronomer's Telegram, 1051,1

Markwardt, C. B., Altamirano, D., Strohmayer, T. E., \& Swank, J. H. 2009, The Astronomer's Telegram, 2237, 1

Pandharipande, V. R., \& Ravenhall, D. G. 1989, in Nuclear Matter and Heavy Ion Collisions, ed. M. Soyeur, H. Flocard, B. Tamain, \& M. Porneuf, NATO ASIB Proc., 205, 103

Papitto, A., Menna, M. T., Burderi, L., di Salvo, T., \& Riggio, A. 2008, MNRAS, 383,411

Papitto, A., Riggio, A., di Salvo, T., et al. 2010, MNRAS, 407, 2575

Papitto, A., Riggio, A., Burderi, L., et al. 2011, A\&A, 528, A55

Patruno, A. 2010, ApJ, 722, 909

Patruno, A., Altamirano, D., \& Messenger, C. 2010, MNRAS, 403, 1426

Rappaport, S. A., Fregeau, J. M., \& Spruit, H. 2004, ApJ, 606, 436

Riggio, A., Papitto, A., Burderi, L., et al. 2011, A\&A, 526, A95

Spitkovsky, A. 2006, ApJ, 648,

Swank, J. H., Markwardt, C. B., \& Smith, E. A. 2005, The Astronomer's Telegram, 449, 1

Thorne, K. S. 1980, Rev. Mod. Phys., 52, 299 